\title{
Hydrogen bond synchronized dual activation enables the unified $\beta$-selective O-glycosylation inside a molecular capsule
}

\author{
Authors: Tian-Ren $\mathrm{Li}^{1}$, Fabian Huck ${ }^{1}$, GiovanniMaria Piccini ${ }^{3,4}$ and Konrad Tiefenbacher ${ }^{1,2 *}$.
}

\section{Affiliations:}

${ }^{1}$ Department of Chemistry, University of Basel, Mattenstrasse 24a, 4058 Basel, Switzerland

${ }^{2}$ Department of Biosystems Science and Engineering, ETH Zurich, Mattenstrasse 26, 4058 Basel, Switzerland

${ }^{3}$ Department of Chemistry and Applied Biosciences, ETH Zurich, c/o USI Campus, Lugano, Switzerland

${ }^{4}$ Facoltà di Informatica, Istituto di Scienze Computazionali, Università della Svizzera Italiana (USI), Lugano, Switzerland

*Correspondence to: konrad.tiefenbacher@unibas.ch / tkonrad@ethz.ch.

Abstract: Carbohydrates are of central importance in biology. The selective chemical synthesis of carbohydrates, however, still poses a challenge; particularly, the selective formation of the thermodynamically labile $\beta$-glycosidic bond is difficult and depends on the substrate's substitution pattern. We here demonstrate that a molecular capsule catalyzes the highly challenging selective formation of $\beta$-glycosides independent of the substrate's substitution pattern and configuration. We demonstrate the versatility of the catalyst by synthesizing small to medium sized 1,2-cis, 2-deoxy, and 1,2-trans $\beta$-glycosides in very high selectivity and good yield. The confined space inside the molecular capsule naturally limits the scope concerning the size of reactants. Interestingly, the proposed mechanism involves the synchronized activation of the glycosyl donor and acceptor inside the supramolecular capsule via a relay involving seven hydrogen bonds. Such an activation is known for enzymes, however, to our knowledge, is unprecedented for man-made catalysts.

Carbohydrates play an extremely versatile and important role in nature. Beside their role in energy storage and as structural components of the cell wall, their involvement in biological recognition events is of special interest. ${ }^{1,2}$ However, the selective chemical synthesis of carbohydrates still remains challenging. ${ }^{3-11}$ One main issue is the stereoselective formation of the glycosidic linkage that, in contrast to the other biopolymers, can yield two isomers; the $\alpha$-isomer featuring the glycosidic bond anti to the substituent at C5 (Fig. 1A), and the thermodynamically less stable $\beta$-isomer with its syn relationship. The selective formation of $\beta$-glycosides is especially difficult. ${ }^{12}$ While the glycoside donor (electrophile) with the leaving group in $\alpha$ configuration is readily available stereoselectively due to its thermodynamic stability, its stereoselective conversion is challenging. Conceptually, two different reaction pathways are possible (Fig. 1B): A clean $\mathrm{S}_{\mathrm{N}} 2$ pathway would deliver the $\beta$-product 2 selectively. In contrast, the $S_{N} 1$ pathway with its cationic intermediate 3 would usually lead to $\alpha / \beta$ mixtures. In practice however, the stereoselective substitution at the anomeric carbon is much more complex since a 
wide continuum of mechanisms between these two prototypical cases has been observed. ${ }^{13}$ The exact pathway is influenced by all reaction parameters involved; not only the solvent, concentration, temperature, catalyst/activating agent, and the nucleophile, but also all aspects of the substrate itself: its substitution and the configuration of the substituents, its conformation that is strongly influenced by the protecting groups utilized, and also the leaving group.

Although tremendous progress has been made, usually many different parameters have to be optimized to achieve excellent selectivities. More importantly, a general, substrate independent, $\beta$-selective glycosylation remained elusive. Only very recently, in 2017, the Jacobsen group reported the first unified approach that allows the catalytic $\beta$-selective construction of a wide range of glycosidic linkages, including the challenging cis-1,2-, and 2-deoxy-products. ${ }^{14}$ The macrocyclic bis-thiourea catalyst 4 activates the electrophile (glycosyl chloride) and the alcohol nucleophile simultaneously (Fig. 1C). Detailed kinetic isotope effect measurements were consistent with an asynchronous $\mathrm{S}_{\mathrm{N}} 2$-mechanism. ${ }^{15}$

We here present an alternative unified catalytic approach involving a molecular capsule. It features (1) an, to our knowledge, unprecedented mechanism for man-made catalysts involving a synchronized activation of the nucleophile and the electrophile through a hydrogen bond relay mechanism (Fig. 1D). (2) This mechanism enables the activation of glycosyl chlorides and even fluorides at ambient or slightly elevated temperatures $\left(50^{\circ} \mathrm{C}\right)$, although no strong hydrogen bond donors or acceptors are present. The activation is achieved solely by hydrogen bonding of water molecules and phenol moieties that are part of the molecular container. (3) The catalyst is readily prepared in large quantities ( $>100 \mathrm{~g}$ batches) in one single step, and displays excellent $\beta$ selectivities for cis-1,2-, trans-1,2-, and 2-deoxy-products.

Molecular capsules, first reported in the early $1990 \mathrm{~s},{ }^{16}$ are self-assembled, closed host structures that allow the reversible binding of guest molecules. To some extend they mimic the binding pocket of enzymes, a feature that has been increasingly utilized for performing chemistry or even catalysis inside such defined nanometer-sized cavities. ${ }^{17-28}$ The unusual example in this report demonstrates that the molecular capsule catalyst I can contribute to tackling a major current synthetic challenge. 


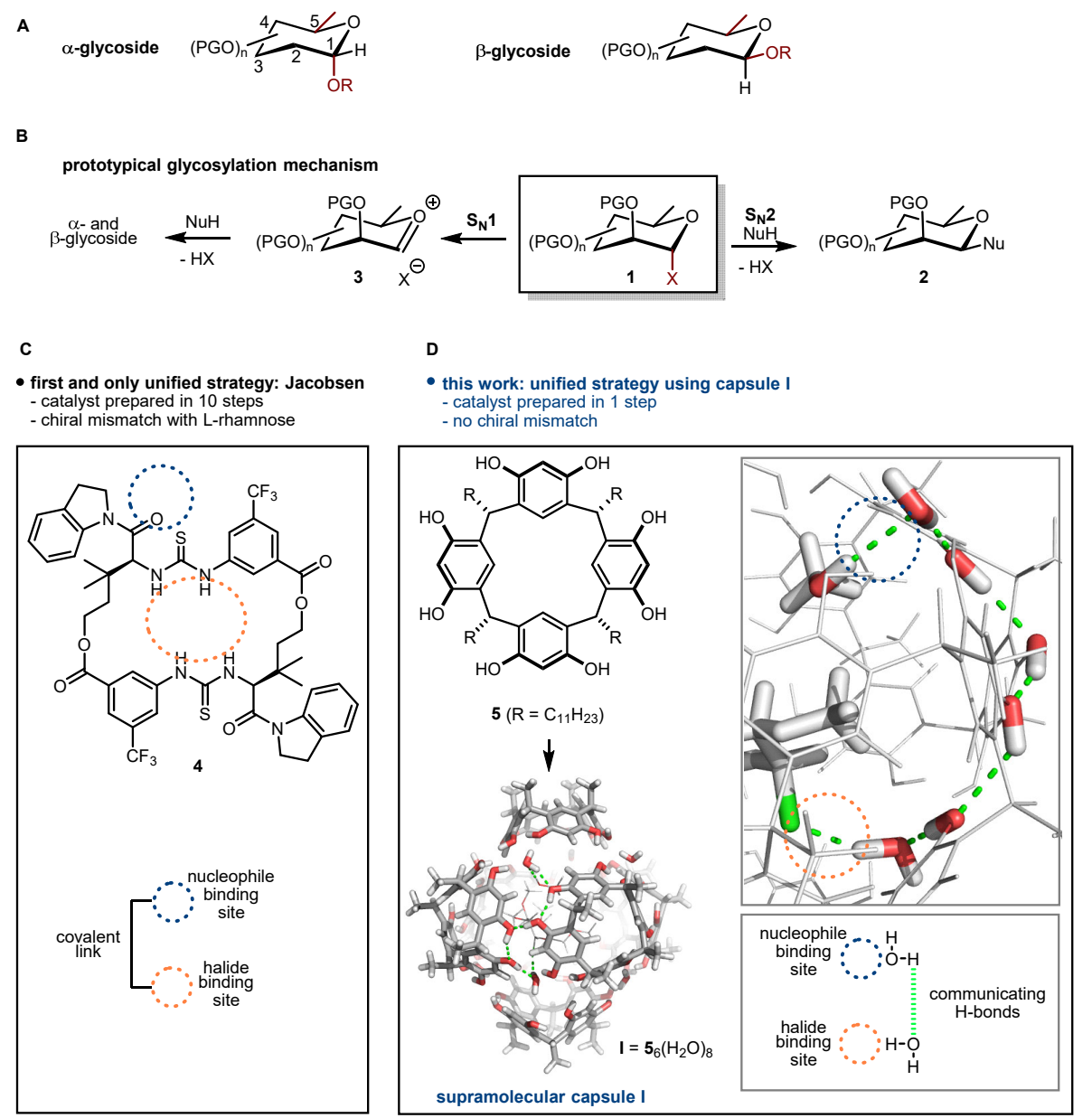

Fig. 1. Overview of selective glycosylations. (A) Glycosides exist in the $\alpha$ - and the thermodynamically less stable $\beta$-form. (B) Glycosylations can be performed by the prototypical $\mathrm{S}_{\mathrm{N}} 1$ or $\mathrm{S}_{\mathrm{N}} 2$ mechanisms. (C) The Jacobsen group reported the first unified approach for the formation of $\beta$-glycosides. The macrocyclic catalyst $\mathbf{4}$ activates the nucleophile and electrophile via strong hydrogen bonds. These binding sites are covalently linked. (D) We report a unified approach based on the readily available supramolecular capsule I as catalyst. Catalyst I selfassembles from six resorcinarene units $\mathbf{5}$ and eight water molecules via sixty hydrogen bonds. The catalyst activates the nucleophile and electrophile, and synchronizes both reaction partners via seven hydrogen bonds. These seven hydrogen bonds are depicted as green dotted lines.

The hexameric capsule I, originally reported by the Atwood group,${ }^{29}$ self-assembles via hydrogen bonds from six resorcinarene units $\mathbf{5}$ and eight water molecules in apolar solvents (Fig. 1D). ${ }^{30-32}$ The first examples for catalysis inside $\mathbf{I}$, either via encapsulation of a catalyst or by the capsule itself, were reported by the Scarso group. ${ }^{33,34}$ Subsequently, our group ${ }^{24}$ and others ${ }^{26}$ utilized the capsule for the catalysis of a variety of reactions. Although $\mathbf{I}$ itself exhibits decent Bronsted acidity (pKa approx. 5.5), ${ }^{35}$ a strong Bronsted acid co-catalyst $(\mathrm{HCl})$ is required for several reactions. ${ }^{36}$ However, even in these cases, the reactions take place inside the cavity, presumably via a proton shuttle mechanism. ${ }^{37}$ Inspired by a report from Aoyama ${ }^{38}$ in 1990 on the 
formation of methyl $\beta$-ribofuranoside from ribose using superstoichiometric amounts of resorcinarene $\mathbf{5}$, we investigated the possibility of performing stereoselective glycosylation reactions inside capsule $\mathbf{I}$.

\section{Results and discussion}

An initial screening revealed that the chloride leaving group displayed promising reactivity in combination with capsule I. Exposing tetra-O-methyl- $\alpha$-D-glucosyl chloride 6 (Fig. 2A; $0.10 \mathrm{M}$ in chloroform, with neutral aluminium oxide (alox) as $\mathrm{HCl}$ scavenger) to 5 eq. methanol for $8 \mathrm{~h}$ in the presence of $10 \mathrm{~mol} \%$ capsule, delivered the methyl glycoside in $79 \%$ isolated yield and very good $\beta$-selectivity $(\alpha: \beta<5: 95)$. To further optimize the reaction conditions, the challenging permethylated $\alpha$-D-mannosyl chloride (Fig. 2B) was utilized as the glycoside donor (see SI for details). Due to its $C 2$-substituent being in an axial $\beta$-orientation, $\beta$-selective glycosylation is not only electronically but also sterically disfavored. ${ }^{4}$ Interestingly, the same reaction conditions were successful; only the temperature was reduced from $30{ }^{\circ} \mathrm{C}$ to $20^{\circ} \mathrm{C}$. Under these conditions, the product formed in 11:89 $\alpha: \beta$-selectivity and was isolated in good yield (75\%). Even higher selectivities were obtained with the permethylated $\alpha$-mannosyl fluoride as donor (Fig. 2C). Although glycosyl fluorides are very stable donors, ${ }^{39}$ an excellent 7:93 $\alpha$ : $\beta$-selectivity and a good yield $(74 \%)$ were obtained at slightly elevated temperature $\left(50{ }^{\circ} \mathrm{C}\right)$, but otherwise identical reaction conditions.
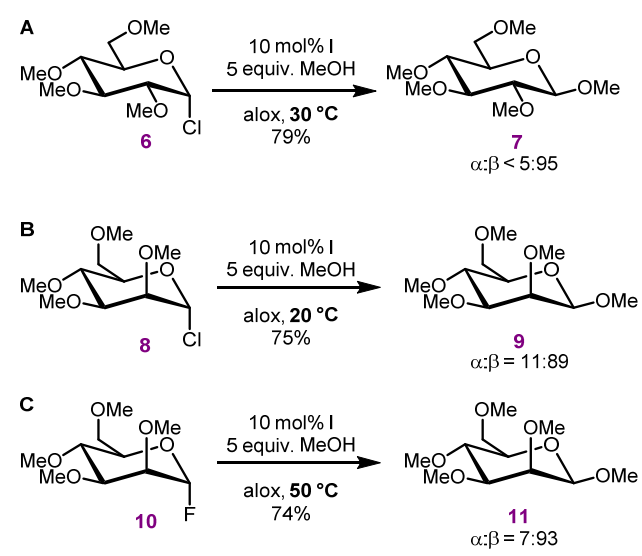

Fig. 2. Summary of screening results. (A) Initial results with glycosyl chloride 6. (B) The same conditions (at a reduced temperature, $20^{\circ} \mathrm{C}$ ) were also applicable to the challenging mannosyl chloride 8 with its $\mathrm{C} 2$-substituent in axial position, which was converted with high selectivity (11:89). (C) An even higher selectivity was observed for the mannosyl fluoride 10 (7:93); for details see SI.

\section{Substrate scope}

With the optimized reaction conditions established, several further nucleophiles were explored (Fig. 3A). Beside primary alcohols, also more hindered secondary alcohols, specifically cyclohexanol and methyl lactate, were explored. For all mannosylations, excellent $\beta$-selectivities were observed, and in most cases the $\alpha$-isomer was barely detectable in the crude reaction mixture by ${ }^{1} \mathrm{H}$ NMR $(\alpha: \beta<5: 95)$. Even more interestingly, the reaction conditions were also 
applicable to alternative glycosyl donors. For instance, L-rhamnosyl fluoride also delivered the $\beta$-products, again a 1,2-cis substitution pattern, in high selectivity. In contrast to the chiral catalyst 4 , which was required in its opposite enantiomeric form to deliver high $\beta$-selectivities for L-rhamnosyl substrates, ${ }^{14}$ in this system no changes to the catalyst were required. The general reaction conditions were furthermore applicable to the 2-deoxy substrates (Fig. 3B). 2-Deoxy substrates are inherently challenging due to the lack of the $\mathrm{C} 2$-substituent able to direct the anomeric selectivity. ${ }^{40}$ For both electrophiles investigated, 2-deoxy-D-glucosyl fluoride and 2deoxy-D-galactosyl fluoride, excellent $\beta$-selectivities and generally very high yields were observed. To complete the electrophile scope, also the formation of 1,2-trans substituted products (16a-e, 17a-e) was explored (Fig. 3C). D-Glucosyl chloride and D-galactosyl fluoride were glycosylated with all five nucleophiles in excellent $\beta$-selectivity and yields. In the case of the D-glucosyl chloride, the match/mismatch situation with the enantiomeric nucleophiles, methyl $(S)$-lactate and $(R)$-lactate, was explored. Both nucleophiles gave excellent $\beta$ diastereoselectivities $(7: 93$ and $<5: 95)$. After having demonstrated the broad scope of glycoside donors, the scope of nucleophiles was extended (Fig. 3D). A whole variety of primary alcohols, carrying sensitive functionalities like an alkyl bromide (16i), epoxide (16k), or an acetal (16) were successfully attached in excellent $\beta$-selectivity. The limitations became visible with the weak nucleophile 2,2,2-trifluoroethanol (16g) that resulted in a reduced yield (32\%) under the standard reaction conditions. A lower yield (34\%) was also obtained for the product containing an epoxide moiety (16k). 


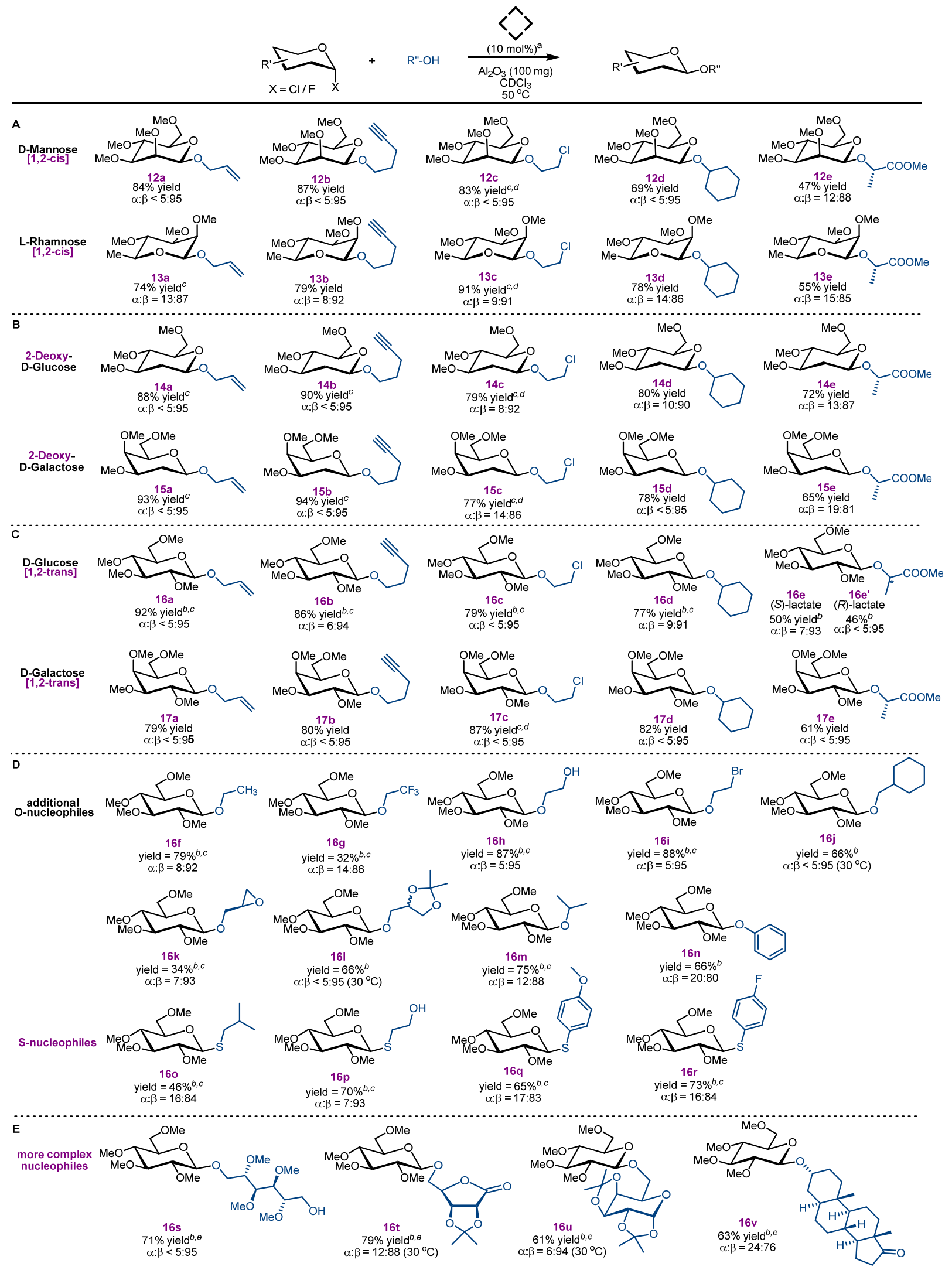


Fig. 3. Substrate table describing the scope of the unified approach for the selective construction of cis-1,2- (A), 2-deoxy- (B), and trans-1,2- (C) $\beta$-glycosidic linkages. ${ }^{a}$ (D) Additional oxygenand sulfur-nucleophiles were explored. (E) Complex nucleophiles were explored that delivered disaccharides in excellent $\beta$-selectivity. ${ }^{a}$ Unless noted otherwise, reactions were performed with glycosyl fluoride (100 $\mu \mathrm{mol}, 1.0$ equiv.), nucleophile (500 $\mu \mathrm{mol}, 5.0$ equiv.), capsule $(66.3 \mathrm{mg}$, $10 \mathrm{~mol} \%)$, alox $(100 \mathrm{mg})$ in $\mathrm{CDCl}_{3}(1.00 \mathrm{~mL})$ at $50^{\circ} \mathrm{C}$; Isolated yields; Diastereomeric ratios were determined by ${ }^{1} \mathrm{H}$ NMR of the reaction mixture. ${ }^{b}$ Glucosyl chloride was used instead of the corresponding fluoride. ${ }^{c}$ Reactions were performed at $20^{\circ} \mathrm{C}$ instead of $50{ }^{\circ} \mathrm{C}$. ${ }^{d}$ alox basic was used instead of alox neutral. ${ }^{e} 3.0$ equivalents of acceptor was used.

Moreover, phenol, thiol, and thiophenol nucleophiles proved to be suitable glycosyl acceptors (16n-16r, Fig. 3D), making this transformation useful for the preparation of known saccharide donors. ${ }^{41}$

Finally, the use of larger, more complex nucleophiles was explored (Fig. 3E). Tetramethyl Dmannitol delivered the disaccharide product 16s in excellent diastereoselectivity. Also 2,3-Oisopropylidene-D-ribonic $\gamma$-lactone, and 1,2:3,4-Di-O-isopropylidene- $\alpha$-D-galactopyranose worked well as nucleophiles, yielding products $\mathbf{1 6 t}$ and $\mathbf{1 6} \mathbf{u}$ in good yields and excellent selectivity. Due to the size limitation inside I, no fully deprotectable disaccharide can be accessed. To explore the size limits of the glycosylation inside the cavity of $\mathbf{I}$, we turned to a steroid nucleophile. Androsterone was converted to the glycoside $\mathbf{1 6} \mathbf{v}$, in slightly reduced $\beta$ selectivity. Molecular modelling (see SI, page 61) indicates that the reagents indeed fill most of the cavity space available. In total, 37 heavy atoms (carbon, oxygen, chlorine) have to be encapsulated to perform this reaction inside the capsule. This is close to the size limitation for reactions inside of $\mathbf{I}$, in agreement with encapsulation studies of tetraalkyl ammonium guests. ${ }^{30}$

After having explored the substrate scope with methylated donors, the use of cleavable protecting groups was investigated (Fig. 4A). Due to the reaction taking place inside the confined space of capsule I (approx. $1400 \AA^{3}$ ), there are obviously limits concerning the reactants' sizes. Nevertheless, both allyl and acetate protecting groups are tolerated very well, providing the products 18a, 18e, 19e, and 20a in good yields and excellent diastereoselectivity ( $\alpha: \beta<5: 95)$. The synthesis of $\beta$-glucoside 18e was also performed on the gram-scale. It was isolated in $85 \%$ yield $(1.09 \mathrm{~g})$ and excellent $\beta$-selectivity, demonstrating the scalability of the procedure. Importantly, catalyst I can be easily recovered by a simple precipitation procedure (see SI part 9 ), and used at least six times without any loss in activity and selectivity. Furthermore, the protecting groups in $\mathbf{1 8 e}$ were cleaved to deliver the unprotected $\beta$-D-glucose derivative 18f. The challenging 2-acetamido substrate substitution pattern was also explored. This pattern usually leads to unreactive oxazoline intermediates, requiring alternative protecting groups for the nitrogen at $\mathrm{C} 2 .{ }^{4}$ In contrast, with the capsule catalyst clean formation of the $\beta$-substituted product 21a in excellent selectivity was observed, further underscoring the versatility of this catalyst system.

\section{Synthesis of known $\beta$-saccharides}

To demonstrate the applicability of the catalyst for the synthesis of known $\beta$-glycosides, two 2deoxy compounds were synthesized. Nonyl 2-deoxy arabinose 22 (Fig. 4B) was chosen as a 
target as it displays mesomorphic behavior, in contrast to its $\alpha$-isomer. ${ }^{42}$ In the literature, it was prepared from a thioglycoside donor that was activated with stoichiometric amounts of Niodosuccinimide and catalytic amounts of trimethylsilyl trifluoromethanesulfonate (TMSOTf), failing to induce stereoselectivity (both isomers were formed in $30-35 \%$ yield). ${ }^{42}$ The methodology presented in this paper yielded a high $\beta$-selectivity (10:90) in the glycosylation of the allyl protected glycosyl fluoride 23 . The product $\mathbf{2 4}$ was obtained in $70 \%$ yield, and was successfully deprotected to deliver the mesomorphic compound 22. More interestingly, the potent anti-tumor agent $\mathbf{2 5}$ (Fig. $4 \mathrm{C})^{43}$ was also readily accessible with the methodology developed. The key step delivered the $\beta$-glycoside 26 in high yield (71\%) and excellent selectivity $(\alpha: \beta=7: 93)$. After acetal cleavage, first the primary and subsequently the secondary alcohols were alkylated to deliver 28. A final deprotection delivered the anti-tumor agent $\mathbf{2 5}$ in excellent overall yield and diastereoselectivity. The synthesis developed compares very favourably to the literature route that relies on the anchimeric assistance of a 2-acetoxy group for $\beta$-selectivity. The required acetoxy group had to be removed subsequently in a lengthy procedure (three steps), leading to a long synthetic sequence of ten linear steps, and fifteen total steps. ${ }^{43}$ The methodology presented here delivered 25 in only eight total steps.

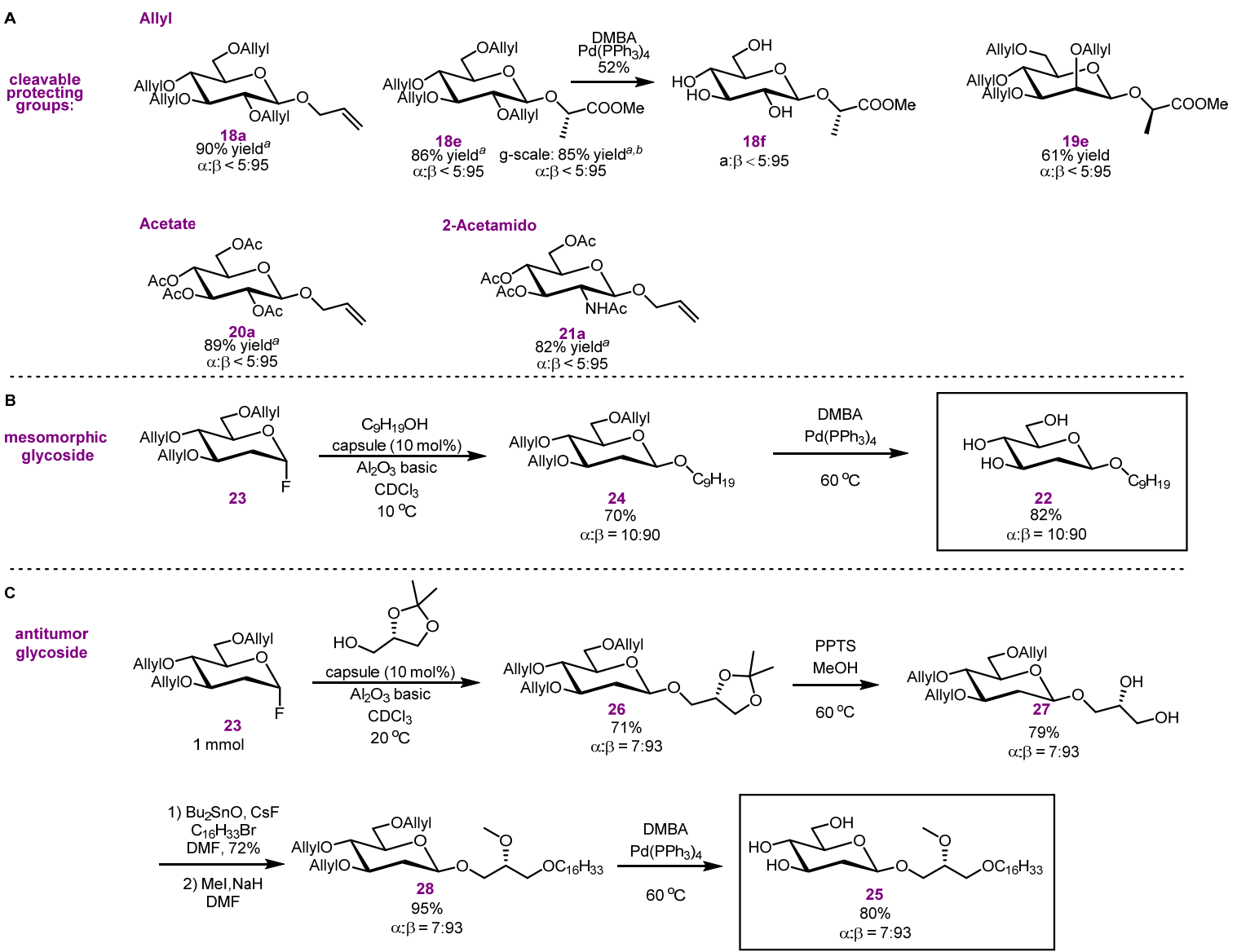

Fig. 4. Substrate table describing examples for fully deprotectable glycoside products. (A) The allyl group and the acetate protecting groups were utilized successfully. (B) Application of the methodology for the $\beta$-selective synthesis of the mesomorphic glycoside 22. (C) Application of the methodology for the $\beta$-selective synthesis of the anti-tumor agent 25 . ${ }^{a}$ The reactions were 
performed with glycosyl chloride (100 $\mu \mathrm{mol}, 1.0$ equiv.), nucleophile ( $500 \mu \mathrm{mol}, 5.0$ equiv.), capsule $(66.3 \mathrm{mg}, 10 \mathrm{~mol} \%)$, alox $(100 \mathrm{mg})$ in $\mathrm{CDCl}_{3}(1.00 \mathrm{~mL})$ at $50{ }^{\circ} \mathrm{C} ;{ }^{b}$ large scale reaction was performed in $\mathrm{CHCl}_{3}$. Isolated yields; Diastereomeric ratios were determined by ${ }^{1} \mathrm{H} \mathrm{NMR}$ of the reaction mixture. DMBA: 1,3-dimethylbarbituric acid.

\section{Control experiments}

The unusually high $\beta$-selectivity for a wide range of differently substituted substrates strongly indicates that the glycosylation takes place inside the cavity of $\mathbf{I}$. In solution such a selectivity would not be expected as extensive research has demonstrated. ${ }^{3-11}$ Nevertheless, a series of control experiments were performed to learn more about the role of the capsule catalyst. Blocking the cavity of $\mathbf{I}$ with an excess of a high affinity guest $\left(\mathrm{Et}_{4} \mathrm{NBF}_{4}\right),{ }^{34}$ efficiently prevented the glycosylation reaction from taking place inside the capsule (Fig. 5A, entry 2). Only small amounts of a diastereomeric product mixture $(\alpha / \beta=44: 56)$ were formed, presumably mainly outside of $\mathbf{I}$. Disassembling the catalyst, either via the addition of DMSO (entry 3 ) or by the use of acetone as a polar solvent (entry 4), prevented product formation in good selectivity, or even completely. The same was true when capsule I was replaced by the closely related hexameric pyrogallolarene (PG) capsule, ${ }^{44}$ known to be unable to catalyze reactions inside its cavity (entry 5). ${ }^{45}$ Also attempts of replacing I with a structural subunit, 4-hexylbenzene-1,3-diol, unable to assemble to a capsule, failed to reproduce the high yields and selectivities (entry 6). The same was true when replacing the mildly acidic capsule catalyst $\mathbf{I}$ with Bronsted or Lewis acids under otherwise identical reaction conditions. Even in larger excess $(200 \mathrm{~mol} \%)$, only a trace of conversion was observed with acetic acid (entry 7) or trifluoroacetic acid (entry 8). The use of superstoichiometric amounts of boron trifluoride led to an acceptable conversion (51\% yield, entry 9), however, like the other acids employed, failed to induce diastereoselectivity.

All results obtained are consistent with the $\beta$-selective glycosylation taking place inside the cavity of catalyst $\mathbf{I}$. If the reaction takes place inside the closed cavity, a substrate size selectivity should be obtainable. Therefore, size competition experiments were performed. In the first set of experiments, the $\alpha$-configured glucosyl chloride 6 was reacted with a 1:1-mixture of differently sized nucleophiles (methanol and 1-docosanol, Fig. 5B). Under standard capsule catalysis, only the methyl glycoside 7 was detectable by ${ }^{1} \mathrm{H}-\mathrm{NMR}$, and as expected in perfect $\beta$-selectivity. Under regular solution conditions $\left(200 \mathrm{~mol}_{\%} \mathrm{BF}_{3}\right.$. $\left.\mathrm{Et}_{2} \mathrm{O}, \mathrm{CDCl}_{3}, 50{ }^{\circ} \mathrm{C}\right)$ both nucleophiles reacted, to deliver a product mixture in a ratio 7/29 of approx. 60:40. Both of these products were formed as $\alpha / \beta$-mixtures in ratios of 58:42 and 45:55, respectively. In the second set of experiments, a mixture of differently sized glycosyl donors (6 and 30) was utilized (Fig. 5C). While capsule catalysis delivered the smaller product $\mathbf{1 6 d}$ exclusively, and in perfect $\beta$ selectivity, the solution experiment again delivered mixtures. These experiments provide very strong additional evidence that the high $\beta$-selectivity stems from a reaction inside the capsule and not on the outer surface, or in solution. To the best of our knowledge, there is no alternative manmade catalyst available that selectively glycosylates mixtures of donors or acceptors of similar reactivity that only differ in size. The selectivity observed opens up possibilities in the emerging field of systems chemistry. ${ }^{46}$ 


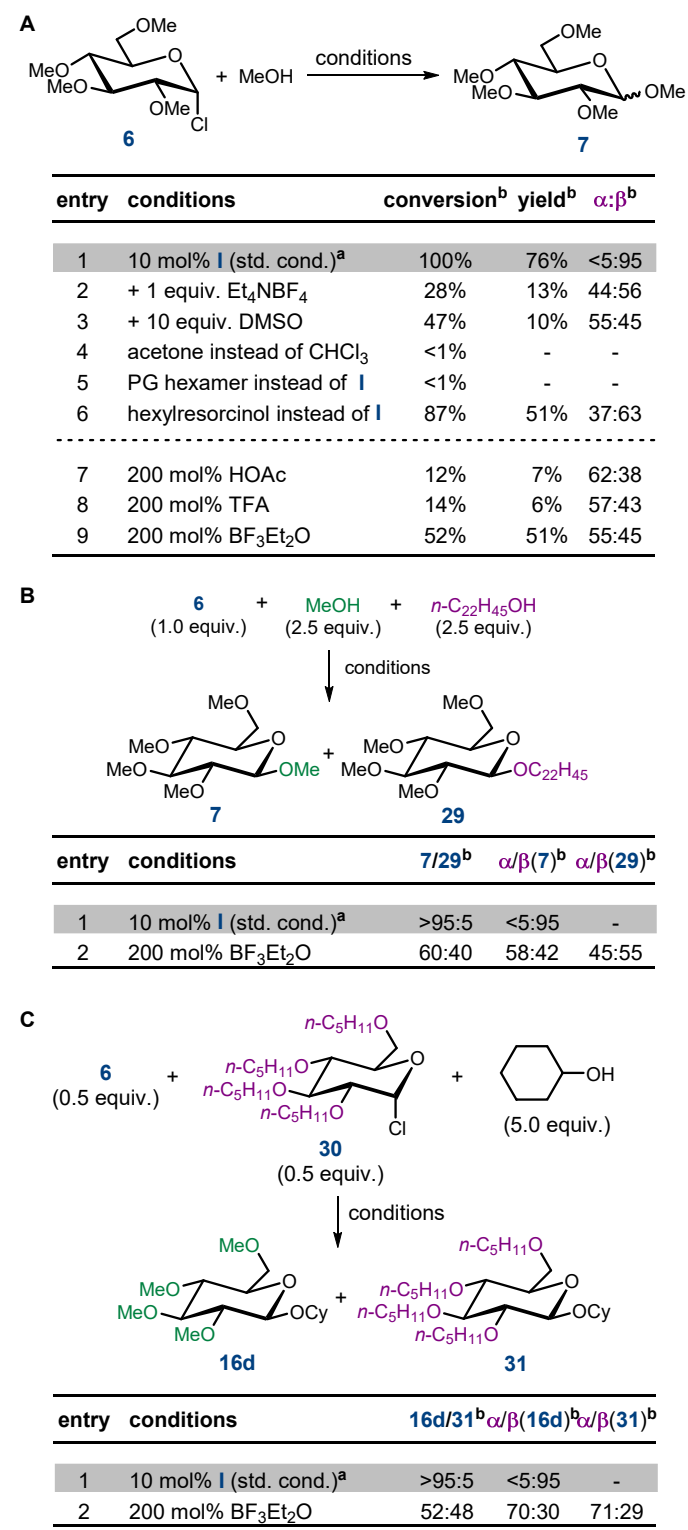

Fig. 5. Control experiments concerning the role of the capsule catalyst. (A) Different reaction conditions that prevent reactions inside the capsule (entries 2-6), and regular solution reactions (entries 7-9) failed to induce high selectivities. (B) A size competition experiment with two different nucleophiles was performed, and indicated that high selectivities can be obtained only inside the capsule catalyst. (C) A size competition experiment with two different donors was performed, indicating that high selectivities can be obtained only inside the capsule catalyst. ${ }^{a}$ standard reaction conditions for glycosyl chloride $\left[10 \mathrm{~mol}^{\%}\right.$ capsule, $\mathrm{CDCl}_{3}, 20^{\circ} \mathrm{C}$, alox as additive]; ${ }^{b}$ Determined by ${ }^{1} \mathrm{H}$ NMR analysis of the reaction mixture.

\section{Mechanistic investigations}

In order to learn more about the glycosylation mechanism inside capsule $\mathbf{I}$, several experiments were performed. First, the dependence of the stereochemical outcome on the electrophile 
configuration was explored. Due to their superior stability, the $\alpha$ - and $\beta$-fluorinated glucosyl donors were prepared in pure anomeric form. While invertive substitution was observed with the $\alpha$-configured glucosyl fluoride $\alpha-32$ (72\% yield, $\alpha: \beta=6: 94$, Fig. $6 \mathrm{~A}$ ), the $\beta$-configured donor $\beta$ 32 delivered an anomeric mixture of the product with a small preference for the $\alpha$-isomer $(54 \%$ yield, $\alpha: \beta=64: 36$, Fig. 6B). Furthermore, kinetic investigations were performed. For the determination of the secondary kinetic isotope effect (SKIE), the deuterated substrate D- $\alpha-32$ (Fig. 6C) was prepared (see SI page 6). A SKIE value of $1.19 \pm 0.021\left(50^{\circ} \mathrm{C}\right)$, corresponding ${ }^{47}$ to a value of $1.21\left(25^{\circ} \mathrm{C}\right)$, was determined from the $\mathrm{H} / \mathrm{D}$ ratios in the isolated product and starting material (see SI, page 75). This value is slightly higher than the SKIE determined by Jacobsen for catalyst $4(1.145 \pm 0.012$ at $\mathrm{rt}) .{ }^{14}$ However, the interpretation of the value is not straightforward when comparing with other literature values. While values of 1.16-1.20 $\left(23{ }^{\circ} \mathrm{C}\right)$ were interpreted as consistent with "an intermediate with oxo-carbonium ion character"; 48 a value very close to the one obtained in our results, $1.19\left(50^{\circ} \mathrm{C}\right)$ was interpreted as consistent with a "loose (exploded) $\mathrm{S}_{\mathrm{N}} 2$ " mechanism. ${ }^{49}$ Therefore, we decided to investigate the reaction order of the nucleophile methanol (Fig. 6D). Interestingly, close to the standard reaction concentration of $0.50 \mathrm{M}$, approx. zero order in methanol was observed. The slightly negative trend likely stems from the destabilization of the capsule via the polar additive methanol. However, at lower methanol concentrations $(0.025-0.100 \mathrm{M})$, a first order in methanol was clearly observed. A first order was also observed for the glycosyl fluoride substrate $\alpha-32$ over the whole concentration range investigated (see SI page 69). The first order in methanol observed excludes a potential $\mathrm{S}_{\mathrm{N}} 1$ mechanism, while the approx. zero order at higher methanol concentrations indicates a saturation of the capsule with methanol, likely via incorporation into the hydrogen bond network. ${ }^{50}$ The very high $\beta$-selectivity, therefore, most likely stems from a loose $\mathrm{S}_{\mathrm{N}} 2-$ mechanism. For the $\alpha$-configured substrate (Fig. 6A), the $S_{N} 2$ inversion takes place efficiently providing very high to quantitative inversion. However, for the $\beta$-configured substrate $\beta$-32 (Fig. $6 \mathrm{~B})$, the $\mathrm{S}_{\mathrm{N}} 2$ mechanism is stereoelectronically disfavored, providing only reduced invertive $\alpha$ selectivity. 


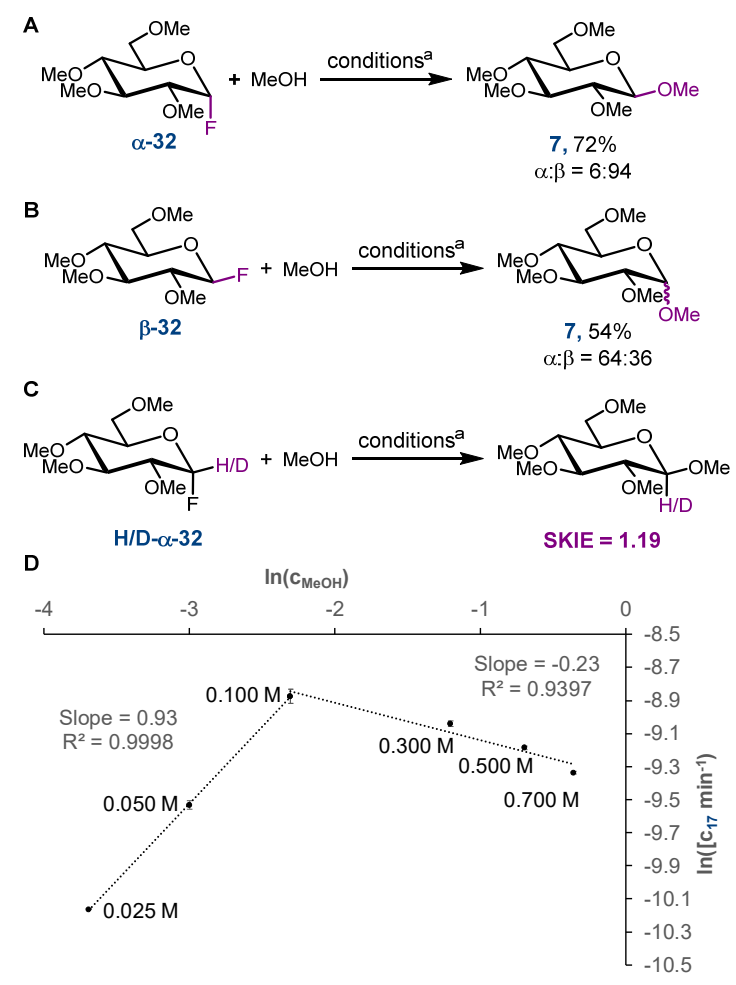

Fig. 6. Mechanistic Investigations. (A) An invertive substitution was observed for the $\alpha$ configured glucosyl fluoride $\alpha-32$. (B) A substantially reduced invertive selectivity was observed for the $\beta$-configured glucosyl fluoride $\beta$-32. (C) The SKIE was determined from a competition experiment of a mixture of regular $\alpha-32$ and its deuterated analog at low conversion (23\%). (D) Logarithmic plot of the initial rate of product formation versus methanol concentration. The slope of the trend line represents the order of the reaction. For methanol concentrations $<0.100$ $\mathrm{M}$ the reactions shows first-order behaviour. Concentrations $>0.100 \mathrm{M}$ lead to a saturation-like behaviour, the negative slope likely stems from a partial disassembly of the capsule at higher methanol concentrations. ${ }^{a}$ standard reaction conditions for glycosyl fluoride [10 mol\% capsule, $\mathrm{CDCl}_{3}, 50{ }^{\circ} \mathrm{C}$, alox as additive]

The unusually mild reaction conditions, devoid of any strong hydrogen bond donor and acceptor, combined with the excellent, and substrate independent, $\beta$-selectivities observed, indicated that

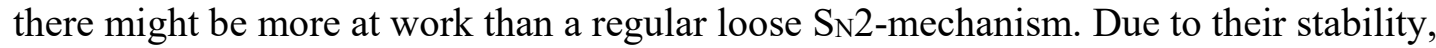
glycoside fluorides usually require strong activation. ${ }^{39}$ However, in the capsule catalyst only hydrogen bonds from water and phenol groups are available. Interestingly, all the hydrogen bonds of $\mathbf{I}$ are communicating, as a proton can be freely shifted over the whole network of 60 hydrogen bonds. This prompted us to postulate a synchronized activation of the electrophile and nucleophile by the hydrogen bond network (Fig. 1D). The electrophile requires a general acid, while the nucleophile depends on a general base for activation. A reciprocal proton transfer enabled via the capsule's hydrogen bond network may satisfy both reaction partners' requirements for activation. 
To learn more about this potential activation mode in atomistic detail, we performed a QM/MM enhanced sampling molecular dynamics (metadynamics) ${ }^{51,52}$ simulation (see SI for details). Figure 7 reports three snapshots of a reactive event during approx. $650 \mathrm{fs}$ of metadynamics simulation time. Prior to the reaction, the chlorine atom is hydrogen bonded to a water molecule at a corner site of the capsule (Fig. 7A) as previously observed for a Friedel-Crafts reaction in a static quantum chemical calculations by Neri, Gaeta, Rescifina, De Rosa, and coworkers. ${ }^{53}$ Here we see that while water donates a proton to the chlorine atom, it begins to abstract another one from an adjacent phenol group. The hydrogen bond network already synchronizes the nucleophile (methanol) and electrophile (glycosyl chloride) through seven hydrogen bonds. After the cleavage of the carbon-chlorine bond (after about 270 fs, Fig. 7B), the hydrogen bond network between the leaving group and the nucleophile strengthens/rigidifies. Although the formation of the new oxygen-carbon bond is fast (approx. further $400 \mathrm{~ms}$, Fig. 7C), it does not occur simultaneously with the rupture of the carbon-chlorine bond. This would suggest a loose $\mathrm{S}_{\mathrm{N}} 2$ transition state in accordance with the experimental findings (see above). After formation of the new oxygen-carbon bond, the excess methanol proton is transferred via the local hydrogen bond network to the water bound to the leaving group.

A

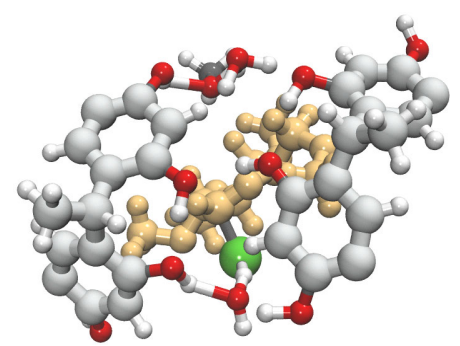

B

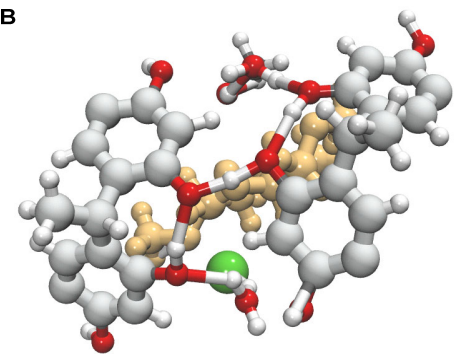

C

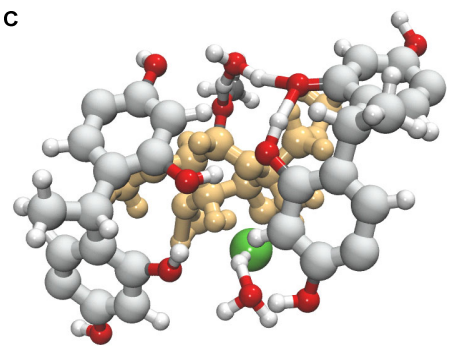

Fig. 7. The three snapshots taken over a span of approx. 650 fs metadynamics simulation time, outline the course of the hydrogen bond synchronized $\mathrm{S}_{\mathrm{N}} 2$ reaction. The structures shown are restricted to the QM region of the calculation. The remaining parts of the capsule, the solvent molecules, and some non-polar hydrogen atoms are omitted for clarity. Color code: the carbon backbone of the resorcinarene backbone (light grey); glycosyl fluoride substrate (yellow); chlorine atom (green); methanol carbon atom (dark grey); oxygen (red); hydrogen (white).

The calculations indicated that indeed a synchronized activation is favoured in this catalyst system. A proton transfer along seven hydrogen bonds (Fig. 1 D) elegantly satisfies the opposing catalytic requirements of the nucleophile and electrophile. Intriguingly, this mechanism is reminiscent of invertive cellulases ${ }^{54}$ that also link a general base and a general acid via a proton relay. Although such mechanisms are known in nature, to our knowledge they have not been reported for man-made catalysts before.

\section{Summary}

A unified approach for the $\beta$-selective construction of cis-1,2-, trans-1,2-, and 2-deoxy glycosidic linkages between various structurally diverse saccharide donors and $\mathrm{O}-$ and Sacceptors was developed. The catalyst is commercially available, or readily prepared on large 
scale in a single step. The glycosylation reaction is scalable without a loss in yield or diastereoselectivity. The reaction conditions are compatible with a wide range of functional groups, including alkenes, alkynes, alkyl chlorides and bromides, esters, alcohols, acetals, allyl ethers, amides, ketones, as well as epoxides. Control experiments provided very strong evidence that the selective glycosylations take place inside the capsule's cavity. Consistent with these results, size selective glycosylation was achieved for the first time with a man-made catalyst. Due to the ready availability of the catalyst and the excellent $\beta$-selectivities obtained for a wide range of donors and acceptors, we expect the methodology to be of great usefulness to the community. As first applications, two known 2-deoxy saccharides with physicochemical or biological functions were efficiently prepared via this methodology. Mechanistic studies, including metadynamics simulations, indicated that the reaction is catalyzed via a hydrogen bond synchronized activation of the nucleophile and electrophile. Although such mechanisms are found in enzymes, this constitutes the first example in a man-made catalyst. This finding will stimulate the discovery of further powerful glycosylation catalysts relying on this activation mode.

\section{References and Notes:}

1 Dwek, R. A. Glycobiology: Toward Understanding the Function of Sugars. Chem. Rev. 96, 683720, doi:10.1021/cr940283b (1996). Varki, A. Biological roles of glycans. Glycobiology 27, 3-49, doi:10.1093/glycob/cww086 (2016).

3 Zhu, X. \& Schmidt, R. R. New Principles for Glycoside-Bond Formation. Angew. Chem. Int. Ed. 48, 1900-1934, doi:10.1002/anie.200802036 (2009).

$4 \quad$ Nigudkar, S. S. \& Demchenko, A. V. Stereocontrolled 1,2-cis glycosylation as the driving force of progress in synthetic carbohydrate chemistry. Chem. Sci. 6, 2687-2704, doi:10.1039/C5SC00280J (2015).

5 Jensen, K. J. O-Glycosylations under neutral or basic conditions. J. Chem. Soc., Perk. Trans. 1, 2219-2233, doi:10.1039/B110071H (2002).

6 Davis, B. G. Recent developments in oligosaccharide synthesis. J. Chem. Soc., Perk. Trans. 1, 2137-2160, doi:10.1039/A809774G (2000). 433, doi:10.1002/open.201600043 (2016).

8 Ling, J. \& Bennett, C. S. Recent Developments in Stereoselective Chemical Glycosylation. Asian J. Org. Chem. 8, 802-813, doi:10.1002/ajoc.201900102 (2019).

9 Nielsen, M. M. \& Pedersen, C. M. Catalytic Glycosylations in Oligosaccharide Synthesis. Chem. Rev. 118, 8285-8358, doi:10.1021/acs.chemrev.8b00144 (2018).

10 McKay, M. J. \& Nguyen, H. M. Recent Advances in Transition Metal-Catalyzed Glycosylation. ACS Catal. 2, 1563-1595, doi:10.1021/cs3002513 (2012). $\mathrm{Li}, \mathrm{W} . \& \mathrm{Yu}, \mathrm{B}$. Gold-catalyzed glycosylation in the synthesis of complex carbohydrate-containing natural products. Chem. Soc. Rev. 47, 7954-7984, doi:10.1039/C8CS00209F (2018). Crich, D. Mechanism of a Chemical Glycosylation Reaction. Acc. Chem. Res. 43, 1144-1153, doi:10.1021/ar100035r (2010). Adero, P. O., Amarasekara, H., Wen, P., Bohé, L. \& Crich, D. The Experimental Evidence in Support of Glycosylation Mechanisms at the SN1-SN2 Interface. Chem. Rev. 118, 8242-8284, doi:10.1021/acs.chemrev.8b00083 (2018). 355, 162-166, doi:10.1126/science.aal1875 (2017). 
Kwan, E. E., Park, Y., Besser, H. A., Anderson, T. L. \& Jacobsen, E. N. Sensitive and Accurate 13C Kinetic Isotope Effect Measurements Enabled by Polarization Transfer. J. Am. Chem. Soc. 139, 43-46, doi:10.1021/jacs.6b10621 (2017). Conn, M. M. \& Rebek, J. Self-Assembling Capsules. Chem. Rev. 97, 1647-1668, doi:10.1021/cr9603800 (1997).

Kang, J. \& Rebek, J. Acceleration of a Diels-Alder reaction by a self-assembled molecular capsule. Nature 385, 50-52 (1997).

8 Yoshizawa, M., Tamura, M. \& Fujita, M. Diels-Alder in Aqueous Molecular Hosts: Unusual Regioselectivity and Efficient Catalysis. Science 312, 251-254, doi:10.1126/science.1124985 (2006).

9 Hong, C. M., Bergman, R. G., Raymond, K. N. \& Toste, F. D. Self-Assembled Tetrahedral Hosts as Supramolecular Catalysts. Acc. Chem. Res. 51, 2447-2455, doi:10.1021/acs.accounts.8b00328 (2018).

Yoshizawa, M., Klosterman, J. K. \& Fujita, M. Functional Molecular Flasks: New Properties and Reactions within Discrete, Self-Assembled Hosts. Angew. Chem. Int. Ed. 48, 3418-3438, doi:DOI 10.1002/anie.200805340 (2009).

Ajami, D. \& Rebek, J. More Chemistry in Small Spaces. Acc. Chem. Res. 46, 990-999, doi:10.1021/ar300038r (2012).

23 Raynal, M., Ballester, P., Vidal-Ferran, A. \& van Leeuwen, P. W. N. M. Supramolecular catalysis. Part 2: artificial enzyme mimics. Chem. Soc. Rev. 43, 1734-1787, doi:10.1039/C3CS60037H (2014).

24 Zhang, Q., Catti, L. \& Tiefenbacher, K. Catalysis inside the Hexameric Resorcinarene Capsule. Acc.

25 Catti, L., Zhang, Q. \& Tiefenbacher, K. Advantages of Catalysis in Self-Assembled Molecular Capsules. Chem. Eur. J. 22, 9060-9066, doi:10.1002/chem.201600726 (2016).

26 Gaeta, C. et al. The Hexameric Resorcinarene Capsule at Work: Supramolecular Catalysis in Confined Spaces. Chem. Eur. J. 25, 4899-4913, doi:10.1002/chem.201805206 (2019).

Morimoto, M. et al. Advances in supramolecular host-mediated reactivity. Nat. Cat. 3, 969-984, doi:10.1038/s41929-020-00528-3 (2020).

Percástegui, E. G., Ronson, T. K. \& Nitschke, J. R. Design and Applications of Water-Soluble Coordination Cages. Chem. Rev. 120, 13480-13544, doi:10.1021/acs.chemrev.0c00672 (2020). MacGillivray, L. R. \& Atwood, J. L. A chiral spherical molecular assembly held together by 60 hydrogen bonds. Nature 389, 469-472, doi:http://www.nature.com/nature/journal/v389/n6650/suppinfo/389469a0 S1.html (1997). Shivanyuk, A. \& Rebek, J. Reversible encapsulation by self-assembling resorcinarene subunits. Proc. Natl. Acad. Sci. U.S.A. 98, 7662-7665, doi:10.1073/pnas.141226898 (2001).

31 Avram, L. \& Cohen, Y. Spontaneous Formation of Hexameric Resorcinarene Capsule in Chloroform Solution as Detected by Diffusion NMR. J. Am. Chem. Soc. 124, 15148-15149, doi:10.1021/ja0272686 (2002). Avram, L., Cohen, Y. \& Rebek, J. Recent advances in hydrogen-bonded hexameric encapsulation complexes. Chem. Commun. 47, 5368-5375, doi:10.1039/c1cc10150a (2011). through encapsulation within a hexameric self-assembled capsule and selective inhibition by a photo-controllable competitive guest. Chem. Commun. 49, 5322-5324, doi:Doi 10.1039/C3cc42233j (2013). 
Cavarzan, A., Scarso, A., Sgarbossa, P., Strukul, G. \& Reek, J. N. H. Supramolecular Control on Chemo- and Regioselectivity via Encapsulation of (NHC)-Au Catalyst within a Hexameric SelfAssembled Host. J. Am. Chem. Soc. 133, 2848-2851, doi:10.1021/ja111106x (2011).

35 Zhang, Q. \& Tiefenbacher, K. Hexameric Resorcinarene Capsule is a Brønsted Acid: Investigation and Application to Synthesis and Catalysis. J. Am. Chem. Soc. 135, 16213-16219, doi:10.1021/ja4080375 (2013).

36 Köster, J. M. \& Tiefenbacher, K. Elucidating the Importance of Hydrochloric Acid as a Cocatalyst for Resorcinarene-Capsule-Catalyzed Reactions. ChemCatChem 10, 2941-2944, doi:doi:10.1002/cctc.201800326 (2018).

Merget, S., Catti, L., Piccini, G. \& Tiefenbacher, K. Requirements for Terpene Cyclizations inside the Supramolecular Resorcinarene Capsule: Bound Water and Its Protonation Determine the Catalytic Activity. J. Am. Chem. Soc. 142, 4400-4410, doi:10.1021/jacs.9b13239 (2020). Tanaka, Y., Khare, C., Yonezawa, M. \& Aoyama, Y. Highly stereoselective glycosidation of ribose solubilized in apolar organic media via host-guest complexation1. Tetrahedron Lett. 31, 61936196, doi:https://doi.org/10.1016/S0040-4039(00)97022-9 (1990). Mukaiyama, T. \& Jona, H. Glycosyl fluoride

A superb glycosyl donor in glycosylation. Proceedings of the Japan Academy, Series B 78, 73-83, doi:10.2183/pjab.78.73 (2002).

40 Bennett, C. S. \& Galan, M. C. Methods for 2-Deoxyglycoside Synthesis. Chem. Rev. 118, 79317985, doi:10.1021/acs.chemrev.7b00731 (2018).

41 Yao, H., Vu, M. D. \& Liu, X.-W. Recent advances in reagent-controlled stereoselective/stereospecific glycosylation. Carbohydr. Res. 473, 72-81, doi:https://doi.org/10.1016/j.carres.2018.10.006 (2019).

42 Singh, M. K., Jayaraman, N., Rao, D. S. S. \& Prasad, S. K. Effect of the C-2 hydroxyl group on the mesomorphism of alkyl glycosides: synthesis and thermotropic behavior of alkyl 2-deoxy-darabino-hexopyranosides. Chem. Phys. Lipids 155, 90-97, doi:https://doi.org/10.1016/j.chemphyslip.2008.07.008 (2008). Marino-Albernas, J. R., Bittman, R., Peters, A. \& Mayhew, E. Synthesis and Growth Inhibitory Properties of Glycosides of 1-O-Hexadecyl-2-O-methyl-sn-glycerol, Analogs of the Antitumor Ether Lipid ET-18-OCH3 (Edelfosine). J. Med. Chem. 39, 3241-3247, doi:10.1021/jm960164j (1996).

44 Gerkensmeier, T. et al. Self-assembly of 2,8,14,20-tetraisobutyl-5,11,17,23tetrahydroxyresorc[4]arene. Eur. J. Org. Chem., 2257-2262 (1999).

45 Zhang, Q., Catti, L., Kaila, V. R. I. \& Tiefenbacher, K. To catalyze or not to catalyze: elucidation of the subtle differences between the hexameric capsules of pyrogallolarene and resorcinarene. Chem. Sci. 8, 1653-1657, doi:10.1039/C6SC04565K (2017).

46 Ashkenasy, G., Hermans, T. M., Otto, S. \& Taylor, A. F. Systems chemistry. Chem. Soc. Rev. 46, 2543-2554, doi:10.1039/C7CS00117G (2017).

47 Crich, D. \& Chandrasekera, N. S. Mechanism of 4,6-O-Benzylidene-Directed $\beta$-Mannosylation as Determined by $\alpha$-Deuterium Kinetic Isotope Effects. Angew. Chem. Int. Ed. 43, 5386-5389, doi:10.1002/anie.200453688 (2004).

48 Matsui, H., Blanchard, J. S., Brewer, C. F. \& Hehre, E. J. Alpha-secondary tritium kinetic isotope effects for the hydrolysis of alpha-D-glucopyranosyl fluoride by exo-alpha-glucanases. J. Biol. Chem. 264, 8714-8716 (1989).

Chan, J., Sannikova, N., Tang, A. \& Bennet, A. J. Transition-State Structure for the Quintessential SN2 Reaction of a Carbohydrate: Reaction of $\alpha$-Glucopyranosyl Fluoride with Azide lon in Water. J. Am. Chem. Soc. 136, 12225-12228, doi:10.1021/ja506092h (2014). 
Slovak, S. \& Cohen, Y. The Effect of Alcohol Structures on the Interaction Mode with the Hexameric Capsule of Resorcin[4]arene. Chem. Eur. J. 18, 8515-8520, doi:10.1002/chem.201102809 (2012).

51 Barducci, A., Bussi, G. \& Parrinello, M. Well-Tempered Metadynamics: A Smoothly Converging and Tunable Free-Energy Method. Phys. Rev. Lett. 100, 020603, doi:10.1103/PhysRevLett.100.020603 (2008).

52 Grifoni, E., Piccini, G. \& Parrinello, M. Microscopic description of acid-base equilibrium. Proc. Natl. Acad. Sci. U.S.A. 116, 4054, doi:10.1073/pnas.1819771116 (2019).

53 La Manna, P. et al. Mild Friedel-Crafts Reactions inside a Hexameric Resorcinarene Capsule: $\mathrm{C}-\mathrm{Cl}$ Bond Activation through Hydrogen Bonding to Bridging Water Molecules. Angew. Chem. Int. Ed. 57, 5423-5428, doi:https://doi.org/10.1002/anie.201801642 (2018).

Nakamura, A. et al. "Newton's cradle" proton relay with amide-imidic acid tautomerization in inverting cellulase visualized by neutron crystallography. Science Advances 1, e1500263, doi:10.1126/sciadv.1500263 (2015).

Acknowledgments: The authors thank Dr. Michael Pfeffer for HR-MS analysis and Prof. Thomas R. Ward for helpful discussions. Molecular dynamics calculations were carried on the ETH Zurich cluster Euler.

Funding: This work was supported by the Swiss National Science Foundation as part of the NCCR Molecular Systems Engineering.

Author contributions: K.T. conceived and supervised the project. K.T. and T.L. planned the project. T.L. carried out all the experiments except the mechanistic investigations concerning SKIE and reaction order, which were performed by F.H. T.L. and K.T. compiled the first draft of the manuscript. GM.P conceived and modelled the simulation of the system, carried out the simulations, interpreted the results, and wrote the first draft of the molecular dynamics section. All authors contributed to the final version of the manuscript.

Competing interests: Authors declare no competing interests.

Data and materials availability: All data is available in the main text or the supplementary materials. Simulations input files will be uploaded on the PLUMED-NEST! repository (https://www.plumed-nest.org/).

\section{Supplementary Materials:}

Materials and Methods

Figures S1-S11, Schemes S1-S8

NMR spectra of isolated compounds 